PACS: $13.66 . \mathrm{Bc}, 13.66 . \mathrm{Jn}$

\title{
CURRENT STATUS OF $e^{+} e^{-} \rightarrow \pi^{+} \pi^{-} \eta$ STUDY. EXPERIMENT AND THEORY
}

\author{
O.N. Shekhovtsova \\ NSC "Kharkov Institute of Physics and Technology" \\ Akademicheskaya, 1, 61108 Kharkov, Ukraine \\ E-mail:shekhovtsova@kipt.kharkov.ua \\ Received November 15, 2017
}

In this note I review the current status of electron-positron annihilation into the $\pi^{+} \pi^{-} \eta$ final state from both experimental and theoretical sides. The theoretical studies of the reaction are discussed in framework of three theoretical approaches: the Vector Meson Dominance Model, the Resonance Chiral Theory with vector and axial-vector resonances and within the extended nonlocal version of the Nambu-Jona-Lasinio model. In the case of the Vector Meson Dominance model the three vector $\rho$ - mesons are included, i.e. the main $\rho(770)$ state and the excited $\rho(1400)$ and $\rho(1700)$ states. For the theoretical approach based on the Resonance Chiral Theory lagrangian the theoretical prediction includes either the lightest $\rho(770)$ state only or the superposition of the main state $\rho(770)$ with the $\rho(1400), \rho(1700)$ states. Finally, the theoretical calculations in the framework of the Nambu-Jona-Lasinio model include both the lightest $\rho(770)$ state and its first excited state $\rho(1400)$. To sum up the theoretical predictions of these three approaches are compared with the recent experimental data from Novosibirsk groups (SND and CMD experiments) and from the BaBar Collaboration. In addition, main points of studies on application of the Conservation Vector Current hypothesis to estimate the cross channel decay width, $\tau^{-} \rightarrow \pi^{-} \pi^{0} \eta \nu_{\tau}$, are discussed. The last subject is an investigation of the OZI and G-parity violated decay $\phi \rightarrow \pi^{+} \pi^{-} \eta$.

KEYS WORDS: precise measurements in electron-positron annihilation, meson production, CVC study, low-energy hadronic interaction

\section{ПОТОЧНИЙ СТАН ДОСЛІДЖЕНЬ ПРОЦЕСА $e^{+} e^{-} \rightarrow \pi^{+} \pi^{-} \eta$. ЕКСПЕРІМЕНТ ТА ТЕОРІЯ}

\section{О.Н. Шеховцова}

ННЦ “Харьковский фізико-техничний інститут”

61108, вул. Академічна, 1, Харків, Україна

У цій статті представлено огляд поточного стану експериментальних і теоретичних досліджень реакції електроннопозитронної анігіляції в кінцевий стан $\pi^{+} \pi^{-} \eta$. Теоретичні дослідження реакції обговорюються в рамках трьох теоретичних підходів: у моделі Векторної Домінантності, в Резонансній Киральной теорії з векторними і аксиально-векторними резонансами, а також в розширеній нелокальній версії моделі Намбу- Йона-Ласиніо. Теоретичні розрахунки у рамках моделі Векторної Домінантності включають вклади від трьох проміжних векторних -мезонов, тобто включені основний стан $\rho(770)$ і збуджені стани $\rho(1400), \rho(1700)$. Теоретичні прогнози грунтовані на лагранжиане Резонансної Кіральної теорії включає в себе або тільки основний стан $\rho(770)$, або суперпозицію основного стану $\rho(770)$ зі збудженими станами $\rho(1400), \rho(1700)$. Нарешті, теоретичні розрахунки у рамках моделі Намбу-Йона-Ласинио включають як основний $\rho(770)$ стан, так і його перший збуджений стан $\rho(1400)$. Резюмуючи, теоретичні прогнози у рамках трьох теоретичних підходів перелічених вище порівнюються з останніми експериментальними даними Новосибірських груп (експерименти SND та CMD) і колаборації BaBar. Крім того, обговорюються основні моменти теоретичних досліджень по вживанню гіпотези збереження векторного струму для оцінки ширини розпаду в крос-каналі $\tau^{-} \rightarrow \pi^{-} \pi^{0} \eta v_{\tau}$. Остання тема даного огляду дослідження рідкісного процесу розпаду $\phi \rightarrow \pi^{+} \pi^{-} \eta$, який відбувається з порушенням O3I і G-парності.

КЛЮЧОВІ СЛОВА: точні вимірювання в електрон-позитронної анігіляції, народження мезонів, дослідження ЗВС, адронна взаємодія при низьких енергіях

ТЕКУЩИЙ СТАТУС ИССЛЕДОВАНИЙ ПРОЦЕССА $e^{+} e^{-} \rightarrow \pi^{+} \pi^{-} \eta$. ЭКСПЕРИМЕНТ И ТЕОРИЯ

О.Н. Шеховцова

ННЦ “Харьковский физико-технический институт"

61108, Академическая, 1, Харьков, Украина

В этой статье рассмотрен текущий статус экспериментальных и теоретических исследований реакции электроннопозитронной аннигиляции в конечное состояние $\pi^{+} \pi^{-} \eta$. Теоретические исследования реакции обсуждаются в рамках трех теоретических подходов: в модели Векторной Доминантности, в Резонансной Киральной теории с векторными и аксиальновекторными резонансами, а также в расширенной нелокальной версии модели Намбу- Йона-Ласинио. Теоретические расчеты в рамках модели Векторной Доминантности включают вклады от трех промежуточных векторных -мезонов, т.е. включены основное состояние $\rho(770)$ и возбужденные состояния $\rho(1400), \rho(1700)$. Теоретические предсказания 
основанные на лагранжиане Резонансной Киральной теории включает в себя либо одно основное состояние $\rho(770)$, либо суперпозицию основного состояния $\rho(770)$ с возбужденными состояниями $\rho(1400), \rho(1700)$. Наконец, теоретические расчеты в рамках модели Намбу-Йона-Ласинио включают как основное состояние $\rho(770)$, так и его первое возбужденное состояние $\rho(1400)$. Резюмируя, теоретические предсказания в рамках трех теоретических подходов перечисленных выше сравниваются с последними экспериментальными данными Новосибирских групп (эксперименты SND и CMD) и коллаборации BaBar. Кроме того, обсуждаются основные моменты теоретических исследований по применению гипотезы сохранения векторного тока для оценки ширины распада в кросс-канале $\tau^{-} \rightarrow \pi^{-} \pi^{0} \eta v_{\tau}$. Последняя тема данного обзора исследование редкого процесса распада $\phi \rightarrow \pi^{+} \pi^{-} \eta$, который происходит с нарушением правила ОЗИ и G-четности.

КЛЮЧЕВЫЕ СЛОВА: точные измерения в электронно-позитронной аннигиляции, рождение мезонов, исследование СВТ, адронное взаимодействие при низких энергиях

The data on the $e^{+} e^{-}$- annihilation in the energy region below 2.-2.5 GeV is the main source of information about properties of the light vector mesons and is of great interest for a better understanding of the light quark interactions. For the process

$$
e^{+}+e^{-} \rightarrow \pi^{+}+\pi^{-}+\eta
$$

the main mechanism of production proceeds through one intermediate state $\eta \rho(770)^{1}$ which makes the precise measurements of the cross section of the reaction (1) of great importance for the determination of the $\rho(1400)$ and $\rho(1700)$ parameters. In addition, the $\pi^{+} \pi^{-} \eta$ gives about $5 \%$ contribution to the total hadronic cross section in the energy region about $1.5 \mathrm{GeV}$ that, in its turn, through the dispersion relation contributes to the anomalous magnetic moment of the muon. Also data on $e^{+} e^{-} \rightarrow \pi^{+} \pi^{-} \eta$ can be used to test the conservation of the vector current (CVC) hypothesis.

The reaction (1) was first observed at the end of 70's in the DM1 experiment [1] and since that time it has been studied by Orsay [2], Novosibirsk [3,4,5,6] groups and by the BaBar collaboration [7,8]. From a theoretical point of view the $\pi^{+} \pi^{-} \eta$ production in the $\mathrm{e}^{+} \mathrm{e}^{--}$- annihilation and $\tau$-lepton decay was discussed in [4,9,10,11] using different approaches.

The paper is organized as follows. In Section "Theoretical Models" the theoretical approaches to describe the hadronic current for $\gamma^{*} \rightarrow \pi^{+}+\pi^{-}+\eta$ are described. The experimental data are reviewed in Section "Experimental data". Section "Check of the CVC hypothesis" is devoted to the CVC study and comparison with the spectral function of the $\tau^{-} \rightarrow \pi^{-} \pi^{0} \eta v_{\tau}$ decay. The subject of the last section is the rare decay $\phi \rightarrow \pi^{+} \pi^{-} \eta$.

Therefore the main goal of this paper is to summarize and systemize the current experimental and theoretical status of the $e^{+} e^{-} \rightarrow \pi^{+} \pi^{-} \eta$ study as well as to show up the main problems of the experimental data analysis of the reaction in the energy region of $2 \mathrm{GeV}$.

\section{THEORETICAL MODELS}

The description of hadronic interactions in the energy region populated by resonances is problematic. In fact, perturbative QCD does not work in the region below $2 \mathrm{GeV}$. At very low energies $\left(E<<M_{\rho}\right)$, Chiral Perturbation Theory (ChPT), based on the chiral symmetry of massless QCD, provides successful description of hadronic interactions. At higher energy there is no a similar framework and we have to rely on some phenomenological models. Three theoretical models have been applied to study the process (1) and they are shortly described below.

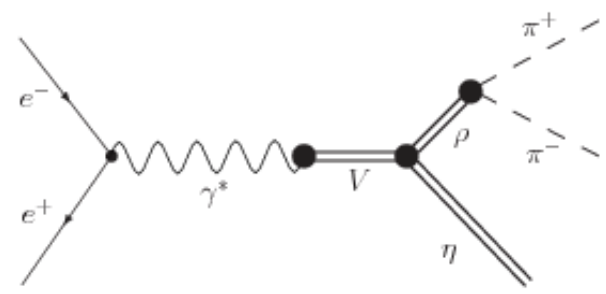

Fig. 1. The diagram of the $e^{+} e^{-} \rightarrow \pi^{+} \pi^{-} \eta$ process in the framework of the VMD model

\footnotetext{
${ }^{1}$ A production via $e^{+} e^{-} \rightarrow a_{2}(1320) \pi$ can contribute to the cross section, however, this reaction is suppressed and has not been observed. To my knowledge there is no theoretical prediction for this reaction as well.
} 
The Vector Meson Dominance (VMD) model with three intermediate $\rho$ meson states is a generally accepted scheme in the contemporary $\pi \pi \eta$ data analysis. It is characterized by the Feynman diagram shown in Fig. 1, where the intermediate resonance $V$ represents the mesons $\rho(770), \rho(1400), \rho(1700)$. The corresponding cross section at the total initial squared energy $s$ can be written as [5]

$$
\sigma(s)=\frac{4 \alpha^{2}}{3} \frac{1}{s \sqrt{s}} \int_{4 m_{\pi}^{2}}^{\left(\sqrt{s}-m_{\eta}\right)^{2}} \frac{\sqrt{q^{2}} \Gamma_{\rho}\left(q^{2}\right) P_{\eta}^{3}\left(s, q^{2}\right)}{\left(q^{2}-m_{\rho}^{2}\right)^{2}+\left(\sqrt{q^{2}} \Gamma_{\rho}\left(q^{2}\right)\right)^{2}}|F(s)|^{2} d q^{2},
$$

where the kinematical function $P_{\eta}^{2}\left(s, q^{2}\right)=\left[\left(s-m_{\eta}^{2}-q^{2}\right)^{2}-4 m_{\eta}^{2} q^{2}\right] / 4 s$ and $q$ is the 4-momentum of the $\pi^{+} \pi^{-}$ system. The transition form factor $F(s)$ describes the subprocess $\gamma^{*} \rightarrow V \rightarrow \eta \rho$ and takes the form:

$$
F(s)=\sum_{V} \frac{g_{V} e^{i \varphi_{V}} m_{V}^{2}}{s-m_{V}^{2}+i \sqrt{s} \Gamma_{V}(s)},
$$

where $g_{V} e^{i \varphi_{V}}=g_{V \rho \eta} / g_{V \gamma}, g_{V \rho \eta}$ and $g_{V \gamma}$ are the coupling constants for the transitions $V \rightarrow \rho \eta$ and $V \rightarrow \gamma^{*}$, respectively. Here the energy dependence of the $\rho$-meson widths is driven by two-pion loops $\Gamma_{V}\left(q^{2}\right)=\Gamma_{V} \frac{m_{V}^{2}}{q^{2}}\left[p_{\pi}\left(q^{2}\right) / p_{\pi}\left(m_{V}^{2}\right)\right]^{3}, p_{\pi}\left(q^{2}\right)=\sqrt{q^{2}-4 m_{\pi}^{2}}$.

Since the coupling constants are not expected to have sizable imaginary parts, the fit to the experimental data is done assuming the phases $\varphi_{\rho(1400)}$ and $\varphi_{\rho(1700)}$ equal either to zero or $\pi$ (if the reference $\rho(770)$ phase is put equal to zero). The SND Collaboration analysis [5] favours $\varphi_{\rho(1400)}=\varphi_{\rho(1700)}=\pi$. Notice that the fit with $\rho(1700)$ is slightly better than without it; however, the value obtained for $g_{\rho(1700)}$ deviates from zero by only $2 \sigma$. Therefore any clear conclusion about the presence of the $\rho(1700)$ state cannot be drawn.

The description of the process (1) within the framework of the Resonance Chiral Theory (RChT) [12] is presented in $[9,10]$. The RChT model is based on the ChPT approach with the explicit inclusion of the vector and axial-vector resonances. Its main features can be summarized in two points: 1) the RChT Lagrangian explicitly contains the ChPT one and hence the RChT amplitudes satisfy chiral symmetry and match the chiral amplitudes at low energies; 2) the high-energy amplitudes of the hadronic form factors provided by RChT are suppressed, as enforced by QCD. Therefore, both high and low energy limits are included correctly in the Resonance Chiral Theory. The $\pi \pi \eta$ production in the RChT framework is described by the diagrams in Fig. 2. Leaving only the diagram 2d), we obtain the VMD approach. In Ref. [9], in addition to the photon propagation, only the lightest $\rho(770)$ resonance was considered.
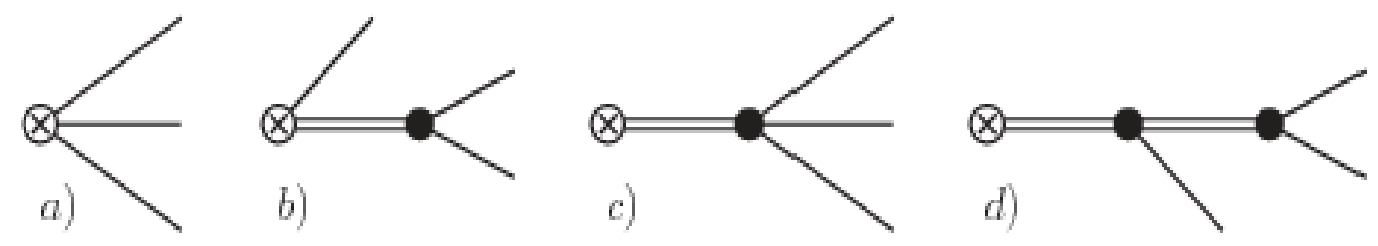

Fig. 2. Hadronization of the vector current in $e^{+} e^{-} \rightarrow \pi^{+} \pi^{-} \eta$ within the RChT approach. Single lines represent pseudoscalar mesons $(\pi, \eta)$ and double lines are for the $\rho$ resonances.

The diagram a) corresponds to the Wess-Zumino-Witten interaction Lagrangian, the diagrams b), c) and d) to the effective Lagrangian describing interactions with resonances

A comparison with experimental data [1-4,7] demonstrates a fair agreement in the energy region from the $\eta \rho$ threshold up to $1.4 \mathrm{GeV}$ (Fig. 3, left panel), for details see [9]. An extended approach of RChT to study the reaction $e^{+} e^{-} \rightarrow \pi^{+} \pi^{-} \eta\left(\pi^{0}\right)$, including the excited $\rho(1400)$ and $\rho(1700)$ mesons as well as tiny isospin symmetry breaking effects, was proposed in [10]. In order to obtain the values of the model parameters, fits based on different sets of $e^{+} e^{-} \rightarrow \pi^{+} \pi^{-} \eta$ and $e^{+} e^{-} \rightarrow \pi^{+} \pi^{-} \eta$ data were carried out. For details, see Section VII of [10]. Comparison between the theoretical prediction for the cross section of process (1) and the experimental data is presented in Fig. 3, right panel. Notice, in addition, that the fit favors an higher mass for the $\rho(1400)$ resonance $\left(M_{\rho^{\prime}}=1.55 \mathrm{GeV}\right.$ instead of its PDG value $\left.M_{\rho^{\prime}}=1.465(25) \mathrm{GeV}\right)$ and lower width $\left(\Gamma_{\rho^{\prime}}=0.238 \mathrm{GeV}\right.$ instead of its PDG value $\left.\Gamma_{\rho^{\prime}}=0.400(60) \mathrm{GeV}\right)$. 
Also the contribution of the $\rho(1700)$ is one half that of the $\rho(1400)$. As in the case of the VMD approximation described above the fit favors to the destructive contributions of both excited $\rho$-states compared with $\rho(770)$.
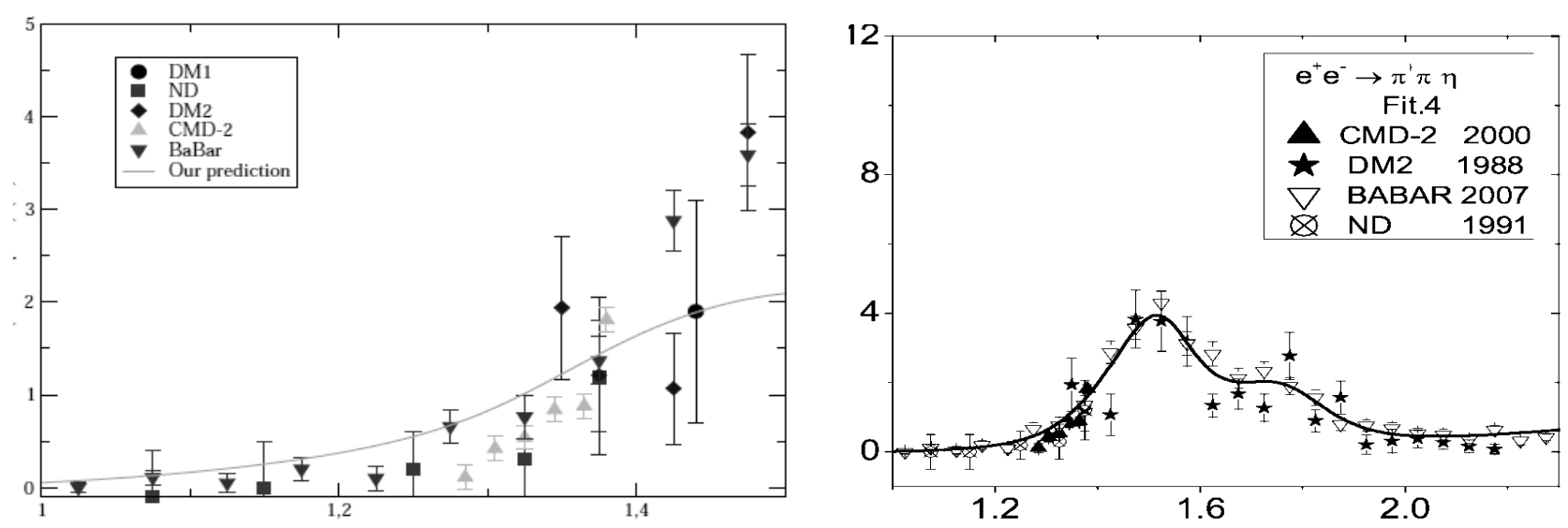

$\sqrt{s}, \mathrm{GeV}$

$\sqrt{s}, \mathrm{GeV}$

Fig. 3. Comparison between the experimental data for the $e^{+} e^{-} \rightarrow \pi^{+} \pi^{-} \eta$ cross section and the theoretical prediction based on the RChT approach

The left panel corresponds to the propagation via $\gamma+\rho(770)$ [9], the right one to the elaborated model used in Ref. [10]. The figures are taken from $[9,10]$

The reaction (1) within the extended nonlocal version of the Nambu-Jona-Lasinio (NJL) model studied in $[11,13]$ and the corresponding Feynman diagrams are shown in Fig. 4. The standard NJL model describes the physical processes via intermediate states of a virtual photon and $\rho$ and $\omega$ mesons in its ground state. The contribution of the first radially excited meson states is included in the extended NJL model and provides a reasonable good description of a wide range of physical processes for the energy region up to about $1.5 \mathrm{GeV}$ [13].
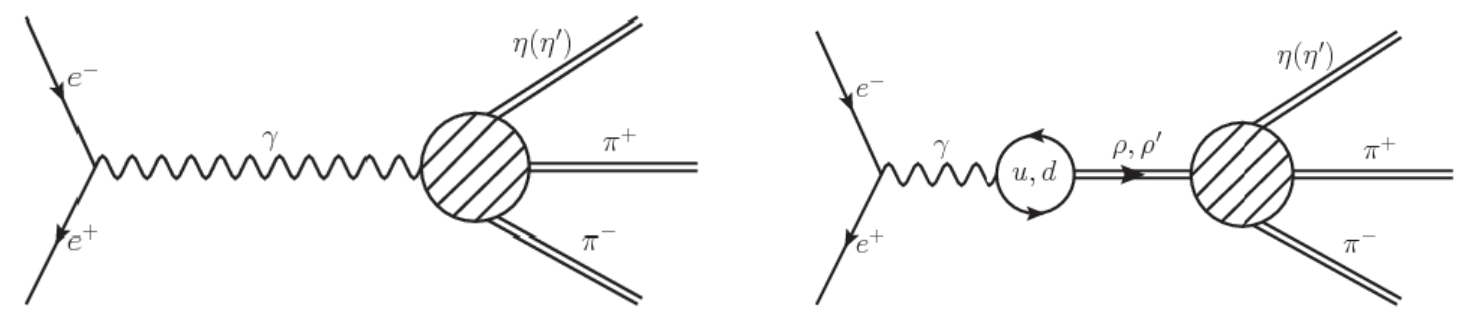

Fig. 4. Electron-positron annihilation with $\pi \pi \eta$ production within the NJL approach

The left diagram corresponds to the production via virtual photon, the right one is via intermediate $\rho(770), \rho(1400)$ states. The dashed circles are the sum of two subdiagrams from Fig. 5.
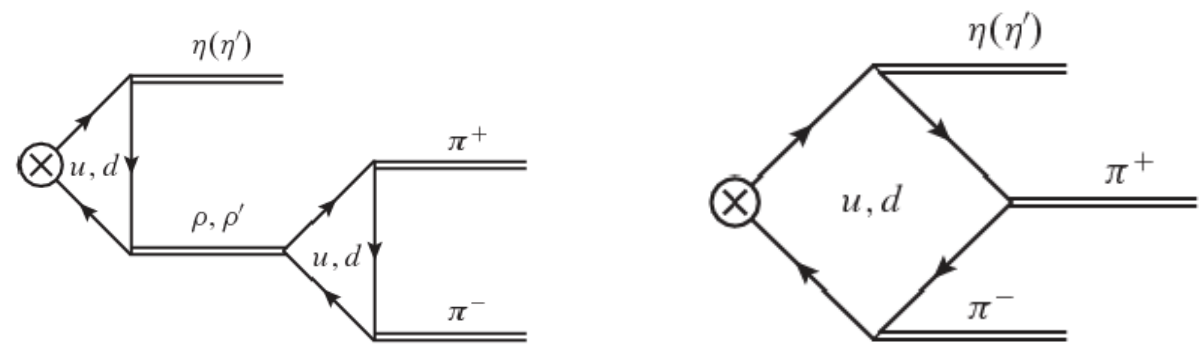

Fig. 5. Vertex subdiagrams $V \eta \pi \pi$ with a quark box loop (left) and with two quark-triangle loops (right).

Hadronization mechanism to produce $\pi \pi \eta$ is presented in Fig. 5. The VMD approach could be directly obtained from the NJL model by taking into account only the diagram with the triangle loops. Precise formulae for the cross section can be found in Section III of [11]. Numerical values of the model parameters are fixed in Eq. (4) of [11] whereas for the resonance masses and widths their PDG values are chosen. The comparison with the BaBar data [7] is 
presented in Fig. 6 and as one can see the theoretical spectrum qualitatively reproduces the experimental data.

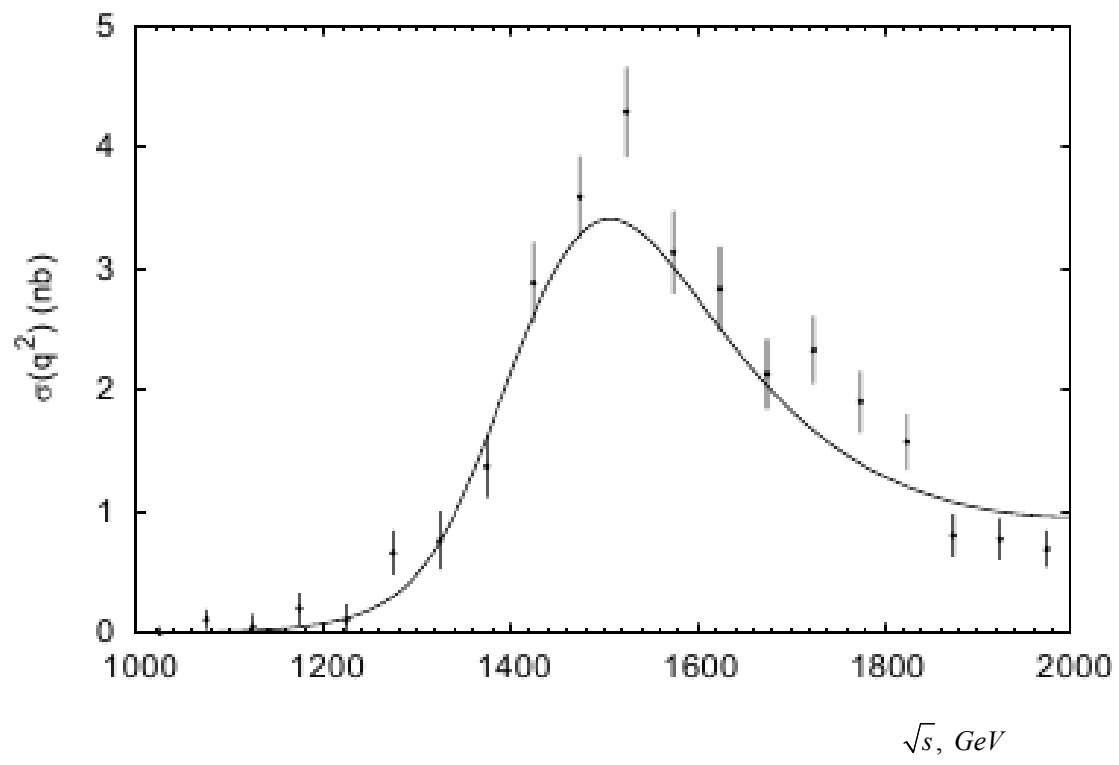

Fig. 6. Comparison of the extended NJL model with the BaBar data [7]. The figure is taken from [11]

\section{EXPERIMENTAL DATA}

As the $\eta$ meson is a short lifetime particle it is observed decaying either in $\pi^{+} \pi^{-} \pi^{0}$ or via its $\gamma \gamma$ decay. Therefore the process (1) contributes to the experimentally measured reactions: $e^{+} e^{-} \rightarrow 2\left(\pi^{+} \pi^{-}\right) \pi^{0}$ and $e^{+} e^{-} \rightarrow \pi^{+} \pi^{-} \gamma \gamma$.

The process (1) was first observed at the end of the 70's in the DM1 experiment [1] ${ }^{2}$ for the center-of-mass (CM) energy region 1.4-2.8 GeV. The DM1 experiment data analysis demonstrated that the $\rho \eta$ production only via the intermediate $\rho(770)$ resonance [16], predicts a cross section of about $0.5 \mathrm{nb}$, an order of magnitude below the experimental data. To solve the problem, the production via the intermediate $\rho(1600)$ was added. The ND Collaboration [3] studied the process in the CM energy region 1.05-1.40 GeV. The data analysis showed the same excess of production compared with the theoretical prediction [15] for energies above $1.35 \mathrm{GeV}$ (Fig. 7), confirming the hypothesis that the main contribution in this energy region comes from the intermediate $\rho(1600)$ meson.

Further comparison of the $\eta \rho$ mass spectrum in diffractive photoproduction [16] and $e^{+} e^{-}$-annihilation has brought to the idea of the existence of two excited states of the $\rho$-resonance with a mass of $1.47 \mathrm{GeV}$ and $1.70 \mathrm{GeV}$, see [17]. This parametrization (the VMD model) has been described at the beginning of Section "Theoretical Models" and since the DM2 experiment it is the generally accepted pattern in the $\pi \pi \eta$ data analysis.

Later the process was studied by Orsay (DM2 Collaboration) [2] and Novosibirsk groups (SND, CMD experiments) [3,4]. The most recent measurements were performed at the VEPP-2000 collider by the SND group [5] for the $\mathrm{CM}$ energy range from 1.22 to $2.00 \mathrm{GeV}$ (the $\eta$ meson is recontructed via the two-photon mode) and at B-factory with the BaBar detector, using the initial state radiation technique [7] in the $(1.025 ; 2.975) \mathrm{GeV} \mathrm{CM}$ energy region (the $\eta$ meson is recontructed via three-pion mode). Systematic uncertainties of about $8 \%$ at BaBar [7] and below $8.3 \%$ by the SND experiment [5] were reported. The preliminary results from BaBar using the $\eta \rightarrow \gamma \gamma$ channel [8] and from the CMD-3 collaboration using both $\eta \rightarrow \pi^{+} \pi^{-} \pi^{0}$ and $\eta \rightarrow \gamma \gamma$ decay modes have been presented recently [6].

The recent experimental results are presented in Figs. 3, 7 and as seen all measurements are consistent. The cross section behaves in the following way: it shows a steep rise starting from the $\eta \rho(770)$ threshold, peaking at about 1.5 $\mathrm{GeV}$, and then decreases with increasing energy. The measured $e^{+} e^{-} \rightarrow \pi^{+} \pi^{-} \eta$ cross section was fitted using the VMD model (Section "Theoretical Models") with and without $\rho(1700)$ contribution. Based on the fit results one cannot draw a definite conclusion that at the current statistics the $\rho(1700)$ contribution is needed for the description of the data.

\footnotetext{
${ }^{2}$ In fact the $e^{+} e^{-}$-annihilation into five pions was observed by the M3N collaboration [15], however, the intermediate mechanisms producing the final state were not studied.
} 

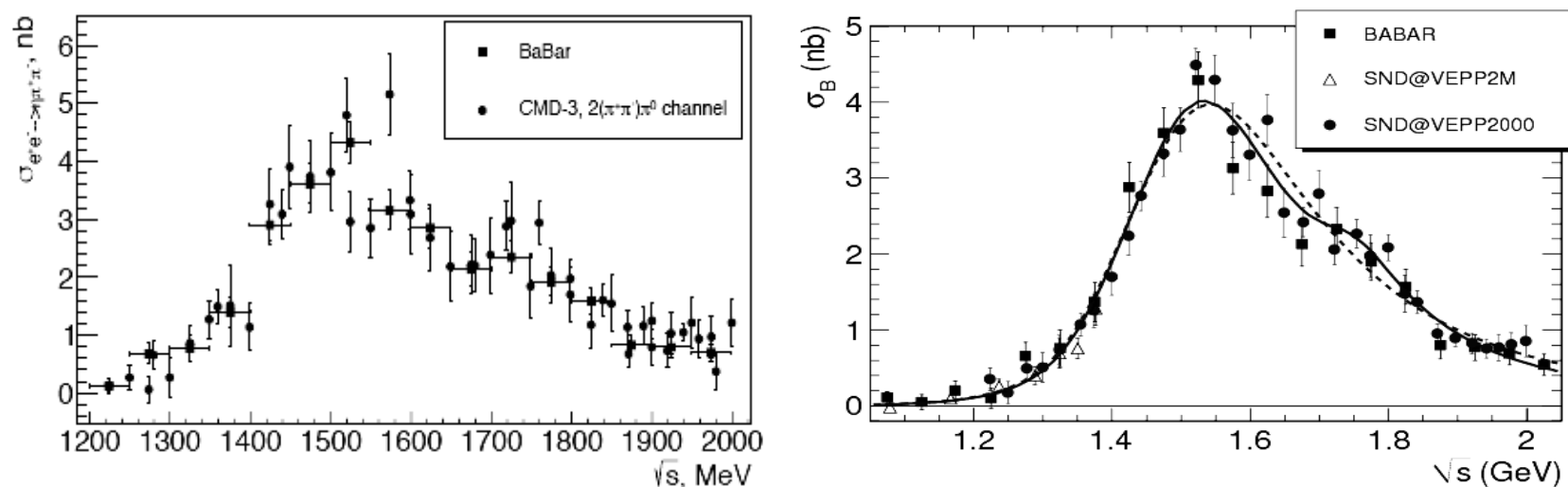

Fig. 7. Experimental data for the $e^{+} e^{-} \rightarrow \pi^{+} \pi^{-} \eta$ cross section from the BaBar, CMD and SND Collaborations

The figures are taken from Refs. [5, 6]

\section{CHECK OF THE CVC HYPOTHESIS}

The data on electron-positron annihilation with $\pi \pi \eta$ production can be used to estimate the branching fraction of the $\tau^{-} \rightarrow \pi^{-} \pi^{0} \eta v_{\tau}$ decay [18] and test of the CVC hypothesis.

The hypothesis of conserved vector current [19] and isospin symmetry is based on two assumptions: first, it assumes that the isovector part of the electromagnetic current and the vector part of the hadronic weak current are only different components of the same isospin current and, second, that the three components of this isospin current are conserved. As a consequence, hadronic currents describing vector $\tau$ decays and hadronic low energy (up to the $\tau$ lepton mass) $e^{+} e^{-}$-annihilation are related and can be obtained one from the other. The CVC hypothesis study has a long story for the two- and four-pion final states [20]. However, a systematical study of CVC relations requires to include all available experimental information on various final states.

Applying the CVC hypothesis for the $\pi \pi \eta$ final state one can relate the experimentally measured cross section of the $e^{+} e^{-} \rightarrow \pi^{+} \pi^{-} \eta$ process and the branching fraction of the $\tau^{-} \rightarrow \pi^{-} \pi^{0} \eta v_{\tau}$ decay [18]:

$$
\frac{B r\left(\tau^{-} \rightarrow \pi^{-} \pi^{0} \eta v_{\tau}\right)}{B r\left(\tau^{-} \rightarrow v_{\tau} e^{-} \bar{v}_{e}\right)}=\frac{3\left|V_{u d}\right|^{2} S_{E W}}{2 \pi \alpha^{2}} \int_{4 m_{\pi}^{2}}^{\infty} d q^{2} \frac{q^{2}}{m_{\tau}^{2}}\left(1-\frac{q^{2}}{m_{\tau}^{2}}\right)^{2}\left(1+\frac{2 q^{2}}{m_{\tau}^{2}}\right) \sigma_{e^{+} e^{-} \rightarrow \eta \pi \pi}\left(q^{2}\right),
$$

where $S_{E W}$ is an electroweak correction $\left(S_{E W}=1.0194\right)$ and the $V_{u d}$ is the $u d$ element of the Cabibbo-KobayashiMaskawa matrix. The theoretical estimation of $\operatorname{Br}\left(\tau^{-} \rightarrow \pi^{-} \pi^{0} \eta v_{\tau}\right)$ is summarized in the Table. As one can see the branching ratio derived from the $e^{+} e^{-}$CMD-2 and old data is in agreement with the world average experimental value whereas the newer BaBar value is slightly above it. A possible source of this difference might be an yet unknown isospin breaking corrections.

Table

$\operatorname{Br}\left(\tau^{-} \rightarrow \pi^{-} \pi^{0} \eta v_{\tau}\right)$ value obtained on the base of the CVC hypothesis from the $e^{+} e^{-}$data and its average world experimental value (PDG).

\begin{tabular}{|l|c|c|c|c|c|c|c|}
\hline $\begin{array}{l}\text { Experimental } \\
\text { group }\end{array}$ & Old $e^{+} e^{-}$data & BaBar 2007 & $\begin{array}{l}\text { Old } e^{+} e^{-} \text {data } \\
+ \text { BaBar 2007 }\end{array}$ & BaBar 2016 & $\begin{array}{c}\text { BaBar } \\
2007+2016\end{array}$ & CMD-2 & PDG \\
\hline$B r, \%$ & $0.13 \pm 0.015$ & $0.165 \pm 0.015$ & $0.153 \pm 0.018$ & $0.16 \pm 0.009$ & $0.162 \pm 0.008$ & $0.156 \pm 0.004$ & $0.139 \pm 0.007$ \\
\hline Reference & {$[18]$} & {$[18]$} & {$[18]$} & {$[8]$} & {$[8]$} & {$[4]$} & {$[21]$} \\
\hline
\end{tabular}

\section{RARE DECAY $e^{+} e^{-} \rightarrow \phi \rightarrow \eta \pi^{+} \pi^{-}$}

Investigation of the energy dependence of the process (1) cross section for the center mass energy near the $\phi$ meson mass is necessary for the search of the rare $\phi \rightarrow \eta \pi^{+} \pi^{-}$decay. This latter is both the Okubo-Zweig-Iizuka rule suppressed and G-parity violating process. Its branching ratio has been theoretically estimated within VMD: $\operatorname{Br}\left(\phi \rightarrow \eta \pi^{+} \pi^{-}\right)=0.35 \cdot 10^{-6}[22]$ and its influence on the $\eta \pi^{+} \pi^{-}$cross section is shown in Fig. 8. A small peak near the threshold is caused by the rare $\phi \rightarrow \eta \pi^{+} \pi^{-}$decay. For illustrative purposes the low energy region is presented in Fig. 8a. The upper limit was measured by the CMD-2 Collaboration and is $\operatorname{Br}\left(\phi \rightarrow \eta \pi^{+} \pi^{-}\right)<1.8 \cdot 10^{-5}$ with $90 \%$ 
confidence level [23]. Therefore the current experimental upper limit is about 50 times above than its theoretical prediction.

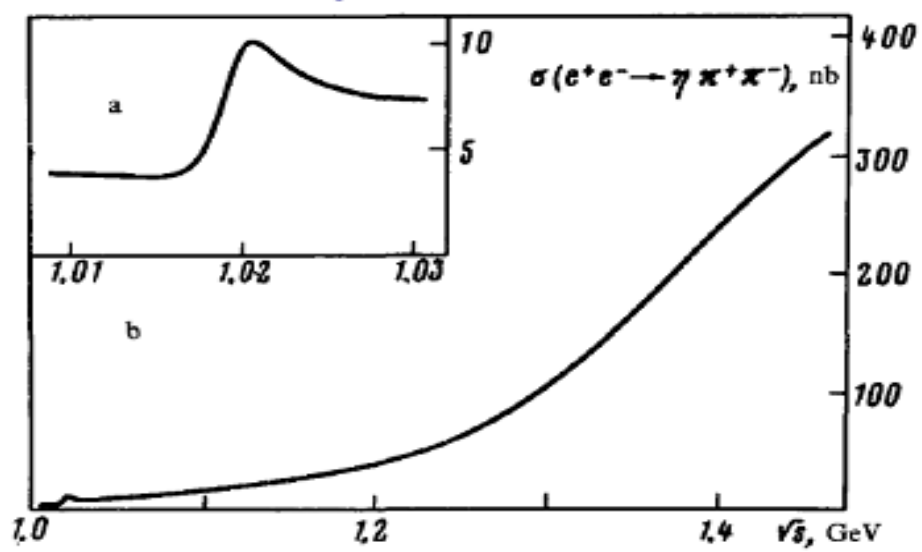

Fig. 8. The VMD prediction for the $e^{+} e^{-} \rightarrow \eta \pi^{+} \pi^{-}$cross section taking into account the rare $\phi \rightarrow \eta \pi^{+} \pi^{-}$decay

The figure is taken from Ref. [22]: $b$ - the whole energy spectrum, a - corresponds to the low energy zoom in to show a small peak due to the rare $\phi \rightarrow \eta \pi^{+} \pi^{-}$decay.

\section{CONCLUSION}

Summarizing, during the last decade the process of $e^{+} e^{-} \rightarrow \pi^{+} \pi^{-} \eta$ has been studied both by theoreticians, using the description based on the Resonance Chiral Lagrangian or the extended Nambu-Jona-Lasinio model, and by experimental collaborations. The data sets at centre mass energies 1.-2. GeV have been collected in $e^{+} e^{-}$-annihilation by the CMD and SND Collaborations (Novosibirsk, VEPP-2M collider) and using the radiative return technique at the SLAC B-factory with the BaBar detector. The systematic uncertainty has been decreased substantially compared with the old experiments and reached the level below $8 \%$ (the preliminary analysis by CMD-3 claims even a $5 \%$ level). The data of the three experiments are in agreement. However, a more detailed study based on the cross section approximation proposed in [18] might be applied for a better comparison between the data in whole energy region.

To fit the measured cross section, the VMD model with three isovector states $\rho(700), \rho(1400)$ and $\rho(1700)$ has been used. However, the current statistics does not allow to conclude whether the $\rho(1700)$ is necessary to reproduce the data. New more precise measurements at energies above $1.4 \mathrm{GeV}$ will be necessary to study the region in detail and to draw a conclusion about the $\rho(1700)$. This study will require an estimate of a background related with the production via $a_{2}(1320)\left(e^{+} e^{-} \rightarrow \rho(1400 ; 1700) \rightarrow a_{2}^{ \pm} \pi^{\mathrm{m}} \rightarrow \pi^{+} \pi^{-} \eta\right)$, but to my knowledge the corresponding process has not yet been calculated theoretically and will be a subject of my future study.

Also new measurements at low energies near the $\phi$ meson region are important to improve our knowledge about the rare decay $\phi \rightarrow \eta \pi^{+} \pi^{-}$(the upper experimental limit is 50 times higher than the theoretical prediction). This is planned to be done at the KLOE-2 experiment.

Assuming the isospin invariance the $e^{+} e^{-} \rightarrow \pi^{+} \pi^{-} \eta$ cross section via the CVC relation predicts the branching ratio $\tau^{-} \rightarrow \pi^{-} \pi^{0} \eta v_{\tau}$. Therefore comparison between the predicted value and its measured value contributes to tests of the $\mathrm{CVC}$ hypothesis. Currently the branching ratio derived from the CMD-2 and old $e^{+} e^{-}$data is in agreement with the average experimental value whereas the predicted value based on the recent BaBar data is slightly above it. A possible source of this difference might be due to not yet calculated isospin-breaking corrections.

Last but not least, the $\pi^{+} \pi^{-} \eta$ contribution gives about $5 \%$ to the total hadronic cross section at energy $\sqrt{s}=1.5$ $\mathrm{GeV}$ and therefore gives a non-negligible contribution to the anomalous magnetic moment of muon, $(g-2)_{\mu}$.

All that makes the reaction $e^{+} e^{-} \rightarrow \pi^{+} \pi^{-} \eta$ an important process of future theoretical and experimental studies.

\section{REFERENCES}

1. Delcourt B., Bisello D., Bizot J.C., Buon J., Cordier A., Mane F. Study of the reactions $e^{+} e^{-} \rightarrow \rho \eta, \rho \pi, \varphi \pi$ and $\varphi \eta$ for total energy ranges between 1.4 and $2.18 \mathrm{GeV} / /$ Phys. Lett. - 1979. - Vol. B 113. - P. 93 - 97.

2. Antonelli A. et al. [DM2 Collaboration]. Measurement of the reaction $e^{+} e^{-} \rightarrow \eta \pi^{+} \pi^{-}$in the center-of-mass energy interval $1350-\mathrm{MeV}$ to $2400-\mathrm{MeV} / /$ Phys. Lett. - 1988. - Vol. B 212. - P. 133 - 138. 
3. Druzhinin V.P. et al.. Investigation of the reaction $e^{+} e^{-} \rightarrow \eta \pi^{+} \pi^{-}$in the energy range up to $1.4 \mathrm{GeV} / /$ Phys. Lett. - $1986 .-$ Vol. B 174. - P. 115 - 117.

4. Akhmetshin R.R. et al. [CMD-2 Collaboration]. Study of the process $e^{+} e^{-} \rightarrow \pi^{+} \pi^{-} \pi^{+} \pi^{-} \pi^{0}$ with CMD-2 detector // Phys. Lett. 2000. - Vol. B 489. - P. 125 - 130.

5. Aulchenko V.M. et al. [SND Collaboration]. Measurement of the $e^{+} e^{-} \rightarrow \eta \pi^{+} \pi^{-}$cross-section in the center-of-mass energy range 1.22-2.00 GeV with the SND detector at the VEPP-2000 collider // Phys. Rev. - 2015. - Vol D 91. - P. 052013.

6. Solodov E.P. et al. Recent results from CMD-3 detector at VEPP-2000 $e^{+} e^{-}$-collider // AIP Conf. Proc. - 2016. - Vol. 1735. P. 020005.

7. Aubert B. et al. [BaBar Collaboration]. The $e^{+} e^{-} \rightarrow 2\left(\pi^{+} \pi^{-}\right) \pi^{0}, \quad 2\left(\pi^{+} \pi^{-}\right) \eta, \quad K^{+} K^{-} \pi^{+} \pi^{-} \pi^{0}$ and $K^{+} K^{-} \pi^{+} \pi^{-} \eta$ Cross Sections Measured with Initial-State Radiation // Phys. Rev. - 2007. - Vol. D 76. - P. 092005.

8. Lees J.P. et al. [BaBar Collaboration]. Study of the process $e^{+} e^{-} \rightarrow \eta \pi^{+} \pi^{-}$using initial state radiation // arXiv: 1801.02960, https://arxiv.org/pdf/1801.02960.

9. Gomez Dumm D., Roig P. Resonance Chiral Lagrangian analysis of $\tau \rightarrow \eta \pi^{-} \pi^{0} v_{\tau}$ decays // Phys. Rev. - 2012. - Vol. D 86. P. 076009.

10. Dai L. Y., Portoles J., Shekhovtsova O. Three pseudoscalar meson production in $e^{+} e^{-}$annihilation // Phys. Rev. - 2013. Vol. D 88. - P. 056001.

11. Volkov M.K., Arbuzov A.B., Kostunin D.G. The process $e^{+} e^{-} \rightarrow \eta\left(\eta^{\prime}\right) 2 \pi$ in the extended Nambu-Jona-Lasinio model // Phys. Rev. - 2014. - Vol. C 89. - P. 015202.

12. Ecker G., Gasser J., Pich A., de Rafael E. The Role of Resonances in Chiral Perturbation Theory // Nucl. Phys. - 1989. Vol. B 321. - P. 311 - 342.

13. Volkov M.K., Arbuzov A.B. Low-energy processes of meson production in the extended Nambu-Jona-Lasinio model // Phys. Part. Nucl. - 2016. - Vol. 47. - P. 489 - 507.

14. Cosme G. et al. Hadronic Cross-sections Study in $e^{+} e^{-}$Collisions From $1.350 \mathrm{GeV}$ to $2.125 \mathrm{GeV} / /$ Nucl. Phys. - 1979. Vol. B 152. - P 215 - 231.

15. Layssac J., Renard F.M. Hadronic production in $e^{+} e^{-}$collisions // Nuovo Cim. - 1971. - Vol. A 6. - P. $134-150$.

16. Atkinson M. et al. [Omega Photon Collaboration]. Observation of a Peak at $1.28-\{\mathrm{GeV}\}$ in the $\eta \pi^{+} \pi^{-}$System in the Reaction $\gamma p \rightarrow \eta \pi^{+} \pi^{-} p$ // Nucl. Phys. - 1984. - Vol. B 242. - P. $269-281$.

17. Donnachie A., Clegg A.B. $\eta \rho$ in diffractive photoproduction and $e^{+} e^{-}$annihilation // Z. Phys. - 1987. - Vol. C 34. P. 257 - 260.

18. Cherepanov V., Eidelman S. Conserved vector current and decays $\tau \rightarrow \eta\left(\eta^{\prime}\right) \pi^{-} \pi^{0} v_{\tau} / /$ JETP Lett. - 2009. - Vol. 89. P. $515-517$.

19. Thacker H.B., Sakurai J.J. Lifetimes and branching ratios of heavy leptons // Phys. Lett. - 1971. - Vol. 36 B. - P. 103 - 105.

20. Davier M., Eidelman S., Hocker A. et al. Confronting spectral functions from $e^{+} e^{-}$annihilation and tau decays: Consequences for the muon magnetic moment // Eur. Phys. J. - 2004. - Vol. C 27. - P. 497- 521.

21. Patrignani C. et al. [Particle Data Group]. Review of Particle Physics // Chin. Phys. - 2016. - Vol. C 40. - P. 100001.

22. Achasov N.N., Karnakov V.A. On the research of the $e^{+} e^{-} \rightarrow \eta \pi^{+} \pi^{-}$reaction // JETP Lett. - 1984. - Vol. $39 .-$ P. 342 - 345.

23. Akhmetshin R.R. et al. [CMD-2 Collaboration]. Observation of the $\phi \rightarrow \pi^{+} \pi^{-} \pi^{+} \pi^{-} / /$Phys. Lett. - 2000. - Vol. B 491. P. 81-89. 